answer questions such as this, the National Geographic Society (Washington, DC, USA) and IBM (Armonk, NY, USA) created the Genographic Project, a five-year research initiative that will trace the migratory history of humans (www.nationalgeographic.com/ genographic/). Under the scientific supervision of population geneticist Spencer Wells, the project will collect mtDNA and Y-DNA data from more than 100,000 indigenous people around the world and an equal number from the general population.

\section{... thestudy of the genetic diversity of parasites and pathogens will providenew insights into human migration patterns if the resultsare integrated with other data...}

O ther attempts to reconstruct human migrations try to piece together information from additional sources. The Journey of Mankind, for example, "a virtual global journey of modern man over the last 160,000 years" presented by the Bradshaw Foundation (Geneva, Switzerland), shows the interaction of human migration and climate (www.bradshawfoundation.com/ journey/). This model is based on the work of Stephen Oppenheimer, a member of Green College at Oxford University, UK, and a fierce supporter of the so-called 'one-source theory'-the hypothesis that modern humans emerged from East Africa in a single migratory wave.

It is quite likely that the study of the genetic diversity of parasites and pathogens will provide new insights into human migration patterns if the results are integrated with other data on our history and if the limitations of this approach are carefully considered (see sidebar). "I think you'll need to look at every microbe on a case-by-case basis," Holmes commented. In addition to answering the question of where we come from and how we got here, knowledge of the evolution and diversity of pathogens might also help us to understand how virulence emerged and varies between different viral, fungal and bacterial strains. Given that diseases have always been closely linked with human movements and evolution, this research might open up new opportunities to develop therapies and preventive strategies against many infectious diseases that afflict specific human populations.

\section{REFERENCES}

Ashford RW (2000) Parasites as indicators of human biology and evolution. J M ed Microbiol 49: 771-772

Cavalli-Sforza LL, Feldman MW (2003) The application of molecular genetic approaches to the study of human evolution. $\mathrm{N}$ at $\mathrm{G}$ enet $\mathbf{3 3}$ : 266-275

Falush D et al (2003) Traces of human migrations in $\mathrm{H}$ elicobacter pylori populations. Science 299: 1582-1585

Fisher M C, Koenig GL, W hite TJ, San-Blas G, N egroni R, Alvarez IG, Wanke B, Taylor JW (2001) Biogeographic range expansion into South America by Coccidioides immitis mirrors N ew World patterns of human migration. Proc N atl Acad Sci U SA 98: 4558-4562

Hector RF, Laniado-Laborin R (2005)

Coccidioidomycosis-a fungal disease of the Americas. PLoS M ed 2: e2

Kittler R, Kayser M , Stoneking M (2003) M olecular evolution of Pediculus humanus and the origin of clothing. Curr Biol 13: 1414-1417

Monot M et al (2005) O $n$ the origin of leprosy. Science 308: 1040-1042
Pavesi A (2005a) U tility of JC polyomavirus in tracing the pattern of human migrations dating to prehistoric times. J Gen Virol 86: 1315-1326

Pavesi A (2005b) M icrobes coevolving with human host and ancient human migrations. J Anthropol Sci 83: 9-28

Reed DL, Smith VS, Hammond SL, Rogers AR, Clayton DH (2004) Genetic analysis of lice supports direct contact between modern and archaic humans. PLoS Biol 2: e340

Shackelton LA, RambautA, Pybus O G, $\mathrm{H}$ olmes EC (2006) JC virus evolution and its association with human populations. J Virol 80: 9928-9933

Wirth T, Wang X, Linz B, N ovick RP, Lum JK, Blaser M, Morelli G, Falush D, Achtman M (2004) Distinguishing human ethnic groups by means of sequences from $\mathrm{H}$ elicobacter pylori: lessons from Ladakh. Proc N atl Acad Sci U SA 101: 4746-4751

Wirth T, M eyer A, Achtman M (2005) Deciphering host migrations and origins by means of their microbes. Mol Ecol 14: 3289-3306

\section{Andre日 Rinaldi}

doi.10.1038/sj.embor.7400908

\title{
A question of faith
}

\section{Exploiting the placebo effect depends on both thesusceptibility of the patient to suggestion and the ability of thedoctor to instil trust}

n $\mathrm{n}$ the same way that physicians have generally had a rather ambivalent attitude towards the relationship between mind and healing, the placebo effect has had a long - and often troubled-relationship with conventional medicine. O ne of the first to describe the power of ineffective medicine was the French philosopher and essayist Michel de Montaigne, who wrote in 1572 "there are men on whom the mere sight of medicine is operative." In fact, in those days, nearly all medicines relied on either some form of placebo effect, or the natural ability of the patients to recover from an illness whether they were treated or not.

Despite being so well-known, it was not until the mid-twentieth century that clinical medicine took notice of the placebo effect. The term itself is often attributed-mistakenly - to the American pharmacologist and anaesthetist Henry Beecher, who had observed how the psychological state of men wounded in the Second World War seemed to influence their perception of pain. In 1955 ,
Beecher wrote, "three-quarters of badly wounded men, although they have received no morphine for hours [... ] have so little pain that they do not want pain relief medication, even though the questions raised remind them that such is available for the asking" (Beecher, 1955). In fact, the term had been used more than three decades earlier in The Lancet (Graves, 1920).

Beecher's observations eventually led him to insist that clinical trials of new drugs could only yield reliable results if conducted in a double-blind fashion-that is, with a control group who were given a placebo without the knowledge of either the patients or the clinicians who administered the drug. Since then, there has been a tendency to regard the placebo effect as background 'noise' that must be subtracted from the results of a trial, rather than as a positive effect that could be exploited clinically.

In general practice, however, doctors widely prescribe placebos and are quite happy to acknowledge this anonymously. In 2004, a study of 89 Israeli physicians found 
that $60 \%$ had used placebos, most commonly to deflect requests for unsuitable medications or in the hope of alleviating undiagnosed but clearly mild conditions (Nitzan $\&$ Lichtenberg, 2004). Of those using the placebo, $68 \%$ told the patient that he or she was receiving real medicine, and $17 \%$ said nothing at all. The remainder either identified the placebo as such (4\%) or told the patient that he or she was receiving a non-specific medicine $(11 \%)$. This raises the ethical dilemma of whether it is appropriate to lie to patientseither tacitly or explicitly-about the nature of their treatment. It seems that for the placebo effect to work, patients need to believe in the treatment. However, their relationship with the doctor is also critical-if patients have faith in the doctor, then even knowingly taking a placebo could be effective.

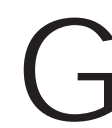

iven mounting evidence that the placebo effect works for some conditions-notably chronic pain and inflammation-researchers and funding agencies are seeking to unravel the mechanisms behind the phenomenon to determine how best to exploit it clinically. The US $\mathrm{N}$ ational Institutes of $\mathrm{H}$ ealth (Bethesda, MD, USA), for example, has made it a priority to identify placebo effects and how they work.

\section{... there has been a tendency to regard the placebo effect as background 'noise' that must be subtracted from the results of a trial, rather than as a positive effect that could beexploited clinically}

The first step in investigating the placebo effect was to document physiological manifestations rather than to accumulate subjective reports of patients 'feeling better'. The most convincing evidence so far comes from the treatment of pain, in which the use of placebos has been found to stimulate the release of endogenous opiates- the body's natural painkillers (Zubieta et al, 2005). However, this discovery did not prove a causal link between placebo, opiate release and pain relief; it was still possible that the placebo could operate in another way, perhaps by conditioning the patient to report a reduction in pain.

The first evidence that a placebo induces a neurobiological effect was found in 1978 when US scientists showed that analgesia, the pain-relieving effect of the placebo, could be blocked by the opioid antagonist naloxone (Levine et al, 1978). The next question was whether a placebo could trigger other biochemical reactions. In the case of pain relief, Martina Amanzio and Fabrizio Benedetti at the University of Turin, Italy, found that a placebo could also reduce pain through non-opioid mechanisms, which could not be blocked by naloxone (Amanzio \& Benedetti, 1999). Benedetti and colleagues have also shown that negative placebo effects, so-called nocebo effects, can cause pain (Benedetti et al, 2006). In addition, placebos have been shown to induce clinical effects against depression through the release of serotonin, against inflammation through the release of cortisol and against Parkinson disease through the release of dopamine (de la FuenteFernández et al, 2002). Benedetti and colleagues have also shown that placebos can induce changes in single neurons in the basal ganglia, which stimulate the release of dopamine and thus relieve symptoms of Parkinson disease (Benedetti et al, 2004).

This begs the question of how to exploit the placebo effect therapeutically. The difficulty is that it is a fickle effect that depends both on the susceptibility of the patient to suggestion and the ability of the doctor to instil belief. Related research into the role of meditation or biofeedback - the attempt to gain conscious control over bodily functions such as heart rate, temperature or blood pressure- sheds light on how patients can induce recovery directly. There is evidence that the practice of meditation can reduce heart rate and body temperature, and alleviate stress. For example, sepsis expert Kevin Tracey at Boston University (MA, USA) is investigating whether biofeedback can bring relief from chronic inflammatory conditions such as Crohn's disease and rheumatoid arthritis. Tracey is trying to exploit his discovery of the inflammatory reflex, whereby the brain senses local inflammation through the vagus nerve, which connects directly with immune cells throughout the body. The brain is able to dampen the immune response by sending messages through the vagus nerve, which blocks the release of pro-inflammatory factors. The failure to constrain this response can lead to chronic inflammatory conditions.

Tracey believes that people can be trained to mitigate the inflammatory response by using biofeedback and is now studying how this can be measured. But he believes that, in the long term, drugs that target the relevant receptors hold greater promise to treat chronic inflammation. The inflammatory reflex seems to operate primarily through nicotinic acetylcholine receptors on tissue macrophages, which are involved in both chronic and acute inflammation, and several groups are now searching for possible drug candidates. "We have shown that you can use muscarinic agonists to increase vagus nerve activity in the periphery, or peripherally acting nicotinic agonists to decrease cytokines in the periphery," said Tracey, referring to recent work (Pavlov \& Tracey, 2006). "These are the two most likely options for fast-track development to activate this pathway with drugs."

$\mathrm{T}$ he placebo effect also manipulates inflammation through the endocrine system, in particular through the release of cortisol by the adrenal glands. This has an important role as it dampens the immune response, raises blood glucose levels and relieves pain by increasing production of endorphins and enkephalins (Sher, 2004). Cortisol can be regarded as the longterm version of adrenaline, which only persists in the blood for a few minutes as a response to an immediate threat-the 'fight or flight' mechanism. Cortisol is metabolized much more slowly and seems to regulate physical activity over a longer time scale, with elevated levels around the time of awakening, for example. It is released in response to what could be called strategic stress, such as an impending exam, rather than immediate danger.

\section{It seems that for the placebo effect to work, the patient needs to believein thetreatment}

The first evidence that a placebo can stimulate cortisol production came by accident more than two decades ago in a study designed to assess the correct dose of ultrasound radiation to relieve pain during dental surgery (Hashish et al, 1986). The findingwhich surprised researchers at the time- was that ultrasound was just a placebo and had no direct therapeutic benefit; however, inflammation was reduced in association with elevated cortisol levels in the region of the tooth. Since then, various experiments and trials have linked the placebo effect with 


\section{The placebo effect is... intended to avoid investing too many resources in an immune response to a relatively minor infection such as the common cold}

cortisol production in other contexts, such as in reducing post-operative swelling.

However, cortisol itself can cause problems as well as solve them. Under stress, the body increases cortisol production, which suppresses all aspects of the immune system, not just inflammation. This can lower resistance to disease and it is possible that relaxation techniques can aid recovery from some infectious illnesses by reducing stress and, at the same time, dampening sympathetic nervous system activity, in turn lowering cortisol production and taking the brakes off the immune system. It might be that the placebo effect and relaxation techniques can help to mediate the production of cortisol.

\section{B} ut if the placebo effect is so beneficial, why do we not invoke it automatically, rather than waiting for an inert pill or a doctor who we trust? According to one of the leading placebo theorists, psychologist Nicholas Humphrey at Cambridge University, UK, the answer could come from research into its evolutionary significance. $\mathrm{He}$ argues that humans have evolved a highly sophisticated health management system designed to control the expenditure of critical resources in order to maximize survival chances. The placebo effect is merely an emergent property of that system, intended to avoid investing too many resources in an immune response to a relatively minor infection such as the common cold. "If somebody has a bacterial illness, then next week comes across another, they would have no resources left if they had thrown everything at the first one," H umphrey explained.

In a similar manner, pain evolved to restrict unnecessary activity and to encourage rest. The placebo effect would then overcome these constraints either when activity becomes necessary or when the associated risk is small-in effect it gives the immune system permission to release the brakes from the body's activities. Seen this way, the placebo effect reverses the antiquated response of the health management system, because the risks against which it provides insurance no longer exist. There is also

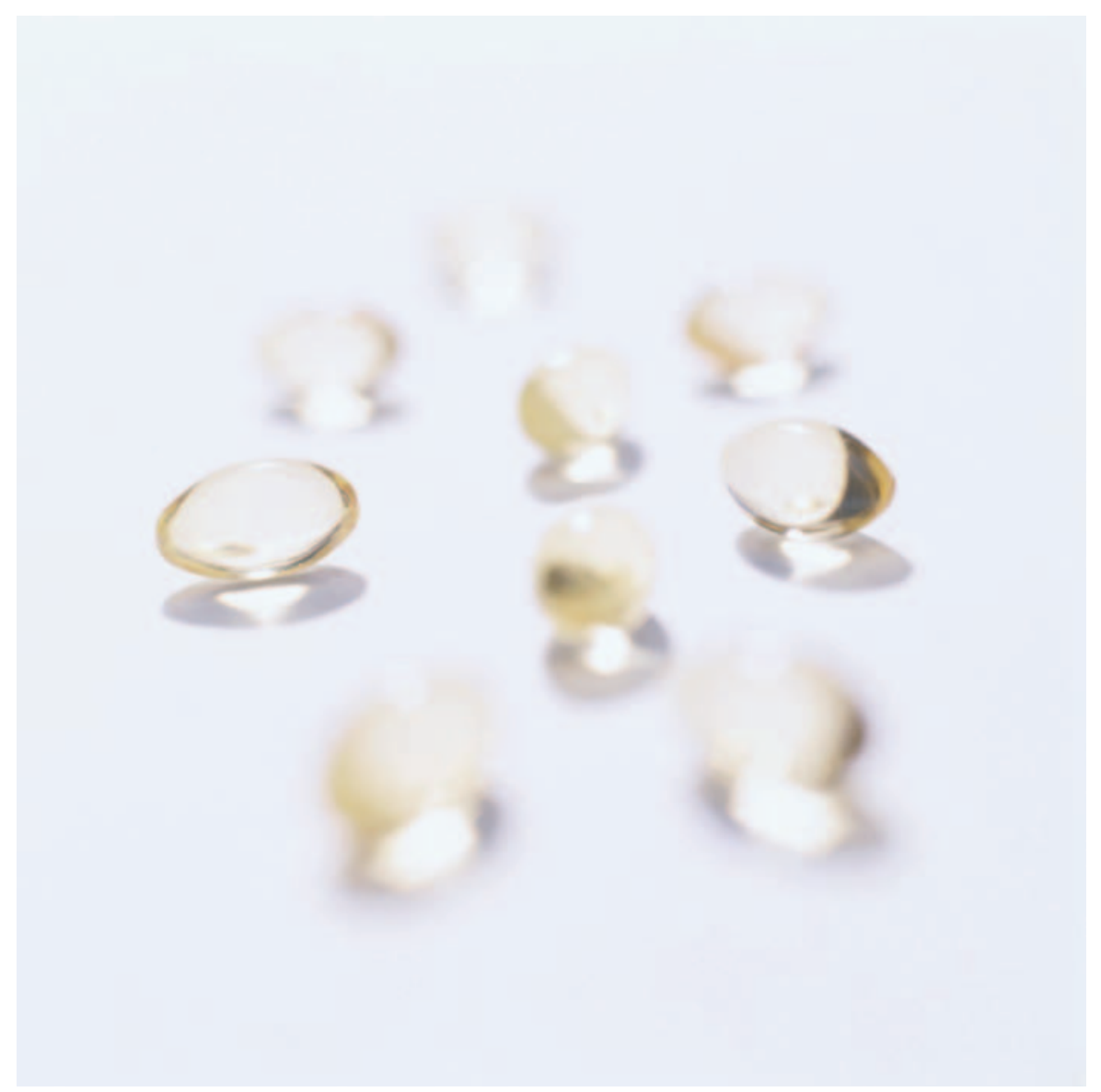

another possibility, although not supported by Humphrey, that feeling ill might itself have an evolutionary function. This would explain why the placebo effect sometimes dampens, rather than stimulates, the immune system - for example, by releasing cortisol into the blood stream. To conserve resources, it might in fact elevate some aspects of the immune response.

\section{Themain problem isthat the placebo effect operates in different ways, with largeindividual and cultural variations}

Benedetti speculates that health management began to evolve in a social context among the leading non-human primates. "This endogenous healthcare system is activated by social contacts with your own social group with the obvious evolutionary advantage that you can recover from a disease and/or improve your quality of life if, and only if, you trust your social group or a single member of the group," he said. "In non-human primates, like chimpanzees, some members of the social group show altruistic behaviours, whereby they take care of sick group-mates." In modern human society, this trusted group member might be a doctor, whereas in ancient societies it was the shaman. "These altruistic chimpanzees represent the early evolution of shamans and modern doctors," Benedetti added.

This behaviour would have been carried forward by natural selection operating at both an individual and a group level. Individuals who trust a member of the social group, be it a chimpanzee, Homo erectus or H omo sapiens, are better placed than those who do not. At the same time, groups that have an individual in whom other members successfully invest their trust are more likely to survive and prosper. Thus, the role of endogenous healthcare management seems to be to identify when to override natural caution through a combination of risk assessment and reassurance by a trusted group member. The question in medical practice is how to exploit this and reinforce clinical or surgical therapies by reassuring the patient. 
The main problem is that the placebo effect operates in different ways, with large individual and cultural variations. The most important distinction is between placebos that exploit the patient's expectation of success and those that operate through conditioning, because success will often depend on selecting the one most appropriate for the condition. "We showed that placebo effects are mediated by expectations when conscious physiological functions, like pain and motor performance, are involved, whereas they are mediated by conditioning when unconscious physiological processes, such as hormone secretion, come into play," said Benedetti.

Benedetti's study included sufferers of Parkinson disease and healthy volunteers who were told that a drug-actually a placebo comprising saline solutionwould deliver pain relief and increase the production of growth hormone while inhibiting cortisol secretion. The verbal suggestion worked for pain relief but not for hormone secretion. However, the patients were then conditioned by replacing the placebo with sumatriptan, a drug that stimulates growth hormone while inhibiting cortisol. When this was later replaced by the placebo, the same pattern of growth hormone stimulation and cortisol inhibition was observed, suggesting that pre-conditioning-but not suggestion-creates the right environment for placebos to influence hormone secretion. In the case of pain relief, where the patient can observe the effect, the opposite was true (Benedetti et al, 2003).

It is not yet clear how this knowledge of the cause and metabolic consequences of the placebo effect could be exploited clinically to benefit patients. In the end, it might just be a matter of common sense: perhaps the greatest impact in medical practice will not be in misleading patients with the prescription of inert pills, but through greater transparency, with doctors only administering treatment in which they have sufficient confidence. This, in itself, might generate a placebo effect.

\section{REFERENCES}

Amanzio M, Benedetti F (1999)

N europharmacological dissection of placebo analgesia: expectation-activated opioid systems versus conditioning-activated specific subsystems. J N eurosci 19: 484-494

Beecher HK (1955) The powerful placebo. JAM A 159: $1602-1606$

Benedetti F, Pollo A, Lopiano L, Lanotte M, Vighetti S, Rainero I (2003) Conscious expectation and unconscious conditioning in analgesic, motor, and hormonal placebo/nocebo responses. J N eurosci 23: 4315-4323

Benedetti F, Colloca L, Torre E, Lanotte M, M elcarne A, Pesare M, Bergamasco B, Lopiano L
(2004) Placebo-responsive Parkinson patients show decreased activity in single neurons of subthalamic nucleus. N at N eurosci 7: 587-588

Benedetti F, Amanzio M , Vighetti S, Asteggiano G (2006)The biochemical and neuroendocrine bases of the hyperalgesic nocebo effect. I Neurosci 26: 12014-12022

de la Fuente-Fernández R, Schulzer M, Stoessl AJ (2002) The placebo effect in neurological disorders. Lancet N eurol 1: 85-91

Graves TC (1920) Commentary on a case of hystero-epilepsy with delayed puberty: treated with testicular extract. Lancet 196: 1134-1135

Hashish I, Harvey W, Harris M (1986) Antiinflammatory effects of ultrasound therapy: evidence for a major placebo effect. $\mathrm{Br}$ J Rheumatol 25: 77-81

Levine JD, Gordon N C, Fields HL (1978) The mechanism of placebo analgesia. Lancet 2: 654-657

Nitzan U, Lichtenberg P (2004) Q uestionnaire survey on use of placebo. BMJ 329: 944-946

PavlovVA, Tracey KJ (2006) Controlling inflammation: the cholinergic anti-inflammatory pathway. Biochem Soc Trans 34: 1037-1040

Sher $L$ (2004) The role of endogenous opioids in the placebo effect in post-traumatic stress disorder. Forsch Komplementärmed Klass Naturheilkd 11: 354-359

Zubieta JK, Bueller JA, Jackson LR, Scott DJ, Xu X, Koeppe RA, NicholsTE, Stohler CS (2005) Placebo effects mediated by endogenous opioid activity on mu-opioid receptors. J N eurosci 25: 7754-7762

\section{PhilipHunter}

doi:10.1038/sj.embor.7400905 